Johannes Mangei

\title{
Dokumentation im Online-Katalog - der Beitrag der Erschließung zur Restaurierung, Digitalisierung und Identifizierung
}

Zusammenfassung: Metadaten zu Sammlungsgegenständen sind im Bibliotheksbetrieb ebenso notwendig wie selbstverständlich. Dass die Erschließung im Verbundkatalog aber auch wichtige Beiträge zu Projekten der Restaurierung, der Digitalisierung und Identifizierung von historischen Drucken leisten kann, wird gelegentlich übersehen. Dabei eignen sich Katalogdaten - außer für Recherche und Verbuchung - auch dazu, spezifischere Angaben zu den vom Brand betroffenen Beständen zu dokumentieren. Dafür stehen längst etablierte Strukturen zur Verfügung. Der Beitrag spricht sich für die regelwerksbasierte, standardisierte Verzeichnung solcher Sachverhalte innerhalb bestehender Datenbanken aus.

Schlüsselwörter: Katalogisierung, Verbundkatalog, Digitalisierung, Restaurierung, Schadensdokumentation

\section{Documentation in the Online Catalogue - the Contribution of Cataloguing to Restoration, Digitization and Identification}

\begin{abstract}
In librarianship, metadata for items in collections are as necessary as they are well-known. But sometimes the fact is overlooked that data in union catalogues can also contribute to projects of restoration, digitization and identification of historical prints. Catalogue data may be suitable - as well as for searching and charging - also in documenting details of stocks affected by fire. For this matter long-established structures are available. The article advocates a rule-based, standardized cataloguing of facts like these within existing databases.
\end{abstract}

Keywords: Cataloguing, union catalogue, digitization, restoration, documentation of damages

Dr. Johannes Mangei: johannes.mangei@klassik-stiftung.de 


\section{Zur Fragestellung}

Dem vorliegenden Beitrag ${ }^{1}$ liegt die Fragestellung zugrunde, welche Datenbankstrukturen für das Vorhalten von Metadaten in Projekten wie z. B. denen des Weimarer Brandfolgenmanagements ${ }^{2}$ am besten geeignet sind. Es wird untersucht, inwiefern die bibliothekarische Erschließung mit Restaurierungsprojekten zusammenwirken und welchen Beitrag die Erschließung dabei zu den restauratorischen Arbeiten leisten kann. Dazu werden die seit 2004 gewonnenen Erfahrungen der Abteilung Medienbearbeitung der Herzogin Anna Amalia Bibliothek dargestellt. Die Ergebnisse sind auch insofern relevant, als sie auf andere Projekte ganz oder teilweise übertragen werden können. Ausgangspunkt der Überlegungen ist dabei mit der Brandkatastrophe und ihren Folgen eine Situation, für die normalerweise im bibliothekarischen Alltag keine Vorsorge getroffen werden kann. Demzufolge muss mit Instrumenten gearbeitet werden, die rasch verfügbar und im langfristigen Einsatz möglichst nachhaltig sind. Diese Bedingungen erfüllen die Datenbankstrukturen der Verbund- bzw. Bibliothekskataloge, die zwar primär die Grundlage für die alltägliche Erschließung und Verwaltung der Sammlungsgegenstände darstellen und die aus der Perspektive der Benutzung eher die Basis für die Recherche und für den Zugriff auf die Medien sind. Doch sind diese (auch im Notfall bereits vorhandenen) Datenbanken und Kataloge neben ihren Grundfunktionen auch für stark spezialisierte Dokumentationsformen wie solche zu den vom Weimarer Bibliotheksbrand betroffenen Beständen gut geeignet. Obwohl die etablierten Bibliothekskataloge - bei aller berechtigten Kritik an Einzelheiten - grundsätzlich als bekannt und als eingeführt gelten dürfen, wird in verschiedenen Projektzusammenhängen anstelle des Standardinstruments Verbundkatalog auf separate Datenbanken zurückgegriffen, bei denen es sich nicht selten um Insellösungen oder Eigenentwicklungen handelt. Das kann im Einzelfall durchaus sachlich begründet und eine inhaltlich sinnvolle Alternative sein, doch sollte die Wahl einer solchen Spezial-Datenbank nicht von vornherein als einzig richtige oder am besten geeignete Möglichkeit angesehen werden. Statt immer neue Sonderwege einzuschlagen, kann auch bei der Dokumentation von Schadens- und Restaurierungssachverhalten auf das sowohl bei der Eingabeseite als auch auf Nutzerseite akzeptierte Instrument des Online-Katalogs zurückgegriffen werden. Dass in diesem Rahmen regelwerksbasierte, standardisierte Formen

1 Ich danke Brigitte Becker-Ebenau, Michael Knoche, Katja Lorenz, Almuth Märker und Jürgen Weber für ihre Ergänzungen, Hinweise und Korrekturen.

2 Dazu Knoche, Michael: Organisatorische Sofortmaßnahmen nach dem Brand der Herzogin Anna Amalia Bibliothek. In: Bibliothek. Forschung und Praxis 30 (2006) S. 221-225. 
der Verzeichnung vorgegeben sind, stellt einen weiteren Vorteil dar. Dasselbe gilt für die Möglichkeiten von Verlinkungen z. B. zur Gemeinsamen Normdatei (GND) sowie für die Option, direkt auf Digitalisate zu verlinken. Innerhalb dieser bestehenden Strukturen werden also von vornherein schon viel anspruchsvollere Anwendungsmöglichkeiten angeboten als nur Eintragungen für die Suche nach Autor, Werktitel oder Signatur.

\section{Zur Katalogsituation der HAAB nach dem Brand 2004}

Voraussetzung für die hier dargestellte Vorgehensweise ist das Vorhandensein von Katalogdaten zu den betreffenden Beständen im Verbundkatalog. Diese Voraussetzung war seit Dezember 2000 für einen großen Teil der Weimarer Bestände, vor allem für die Alten Drucke, erfüllt. Denn zu diesem Zeitpunkt konnte das von der Deutschen Forschungsgemeinschaft (DFG) geförderte Altbestandserfassungsprojekt zur Überführung der konventionellen Zettelkataloge in den Verbundkatalog erfolgreich abgeschlossen werden. ${ }^{3}$ Zum Zeitpunkt des Brandes im September 2004 lagen diese Daten in den Strukturen des Gemeinsamen Verbundkatalogs (GVK) vor. Dagegen fehlten sie für knapp 10000 Drucke (bzw. 11000 Datensätze), die nach 1850 erschienen und vom Brand betroffen sind. Sie waren im alphabetischen Zettelkatalog erschlossen, dessen Titelkarten sich gerade im Prozess der Konvertierung befanden. Dennoch waren so immerhin die älteren Druckschriften und der größere Teil des alphabetischen Katalogs für die jüngeren Bestände (ab 1851) im Verbundkatalog erfasst. Die Einzelheiten dieser Ausgangslage sind an anderer Stelle bereits ausführlicher beschrieben worden, ${ }^{4}$ so dass hier diese grundsätzlichen Angaben ausreichen.

3 Becker-Ebenau, Brigitte: Das Altbestandserfassungsprojekt der Herzogin Anna Amalia Bibliothek Weimar. Motor für bibliotheksinterne Entwicklungen - ein Erfahrungsbericht. In: Bibliotheksdienst 35 (2001) S. 707-714.

4 Becker-Ebenau, Brigitte: Verlust- und Schadensdokumentation. In: „Es nimmt der Augenblick, was Jahre geben“. Vom Wiederaufbau der Büchersammlung der Herzogin Anna Amalia Bibliothek. Im Auftrag der Klassik Stiftung/Herzogin Anna Amalia Bibliothek hrsg. von Claudia Kleinbub, Katja Lorenz und Johannes Mangei. Göttingen 2007, S. 38-40, und Mangei, Johannes: „Ersatzbeschaffung“ für Verluste durch den Brand der Herzogin Anna Amalia Bibliothek vom 2. September 2004. In: Bibliothek. Forschung und Praxis 30 (2006) S. 56-62. 


\section{Erschließungsformen im Rahmen der Brandfolgeprojekte}

\subsection{Dokumentation der Verluste}

Bereits unmittelbar nach dem Bibliotheksbrand im September 2004 wurde im Rahmen der ersten organisatorischen Sofortmaßnahmen die Grundlage für eine Dokumentation der mutmaßlichen Verluste mittels des Online-Katalogs bzw. anhand der Verbunddatenbank des Gemeinsamen Bibliotheksverbundes (GBV) gelegt: Dazu wurden mit Hilfe eines Verzeichnisses, das sämtliche Standorte der Bücher im Rokokosaal dokumentierte, die Katalogdaten derjenigen Bände, die auf der Zweiten Galerie aufbewahrt wurden (wo das Feuer ausbrach und die größten Schäden hervorrief), pauschal mit Kodierungen und mit der Anmerkung „Vermutlich Verlust bei Bibliotheksbrand 2004“ versehen. Im Zusammenwirken mit der Verbundzentrale des Gemeinsamen Bibliotheksverbundes (VZG) gelang es, innerhalb von 18 Tagen eine Datenbank mit damals ca. 50000 als Verluste gekennzeichneten Titeln in Form einer Teilsicht aus dem Gesamtkatalog freizuschalten. ${ }^{5}$ Dafür wurden keine neuen Katalogisate erstellt, sondern zunächst pauschale Ergänzungen an den vorhandenen Datensätzen vorgenommen. Diese Angaben wurden überwiegend mit Hilfe von Skripten eingetragen, die sich auf die Rechercheergebnisse zu den vom Brand betroffenen Signaturengruppen der Zweiten Galerie des Rokokosaals bezogen. Nur so konnte die große Datenmenge in vergleichsweise kurzer Zeit bewältigt werden. Seither werden die Einträge für jeden Einzelfall, der sich nicht als verbrannt, sondern als Brandschaden oder als wider Erwarten erhaltenes Exemplar herausstellt, entsprechend aktualisiert. So konnten in den ersten Jahren nach dem Brand in diesem Rahmen mehr als 8600 Katalogeinträge entsprechend geändert und um den Zusatz „Unversehrt erhalten“ ergänzt werden. Außerdem wurden später Änderungen bei bisher etwa 6300 Datensätzen aus einem Geschäftsgang vorgenommen, der zur Erschließung, Restaurierung und Digitalisierung der sogenannten „Aschebücher“ seit Juli 2009 etabliert wurde. ${ }^{6}$ Die dabei bearbeiteten Bestände waren unmittelbar nach dem Brand noch als Verluste angesehen worden (dazu ausführlicher unten, Abschnitt 3.3). Als Brandverluste wurden dagegen nachträglich etwa 11000 Datensätze neu dokumentiert, die Bücher mit Erscheinungsjahren nach 1850 betreffen

5 http://opac.ub.uni-weimar.de/DB=2.2/ [Zugriff: 28. März 2013].

6 Vgl. Izdebska, Magdalena: Weimarer Aschebücher. Neue Restaurierungstechnologien in der Mengenrestaurierung. In: Wochenblatt für Papierfabrikation 140 (2012) S. 196-200. 
und nach dem Brand im Rahmen der Katalogkonversion erstmals elektronisch im Verbundkatalog erfasst wurden. Um den Interessen von Lesern, Wissenschaftlern, Antiquariaten, aber auch von hilfsbereiten Sammlern und Bibliotheken entgegen zu kommen, wurde außerdem eine Website erstellt, die vorformulierte Suchen im Weimarer Online-Katalog enthält und damit komplizierte Recherchen nach bestimmten Verlusten auf einen einzigen Mausklick reduziert. ${ }^{7}$ Dabei wurde außerdem dem Wunsch Rechnung getragen, dass Verluste, für die weitere Exemplare vorhanden sind und die deshalb für eine Wiederbeschaffung nicht in Frage kommen, von der Ergebnisanzeige ausgeschlossen werden. Auch diese Anwendung beruht auf den bereits vorhandenen Katalogisaten der Verbunddatenbank.

\subsection{Dokumentation der Einbandschäden}

Nachdem die Dokumentation der Verluste eingerichtet war, bot es sich an, auch die durch Löschwasser, -schaum und Hitzeeinwirkung entstandenen Schäden an Büchern außerhalb des unmittelbaren Brandherdes in derselben Datenbankstruktur, d.h. an den GVK-Datensätzen, zu erfassen. ${ }^{8}$ Eingetragen wurden Angaben zu den leichten Wasserschäden - die Bücher konnten nach der Gefriertrocknung schon früh wieder der Benutzung zugeführt werden - sowie zu unterschiedlich gravierenden Einbandschäden. Dazu wurde zwischen restauratorischer Bestandsaufnahme und bibliothekarischer Erschließung eine enge Abstimmung hergestellt: Die einzelnen, standardisierten Kriterien aus der Schadensbeschreibung der Restauratoren wurden von den bibliothekarischen Mitarbeitern in lokale Normdaten übertragen.

7 http://haab.weimar-klassik.de/brandverluste/ [Zugriff: 28. März 2013].

8 Becker-Ebenau (wie Anm. 4), hier S. 39. 


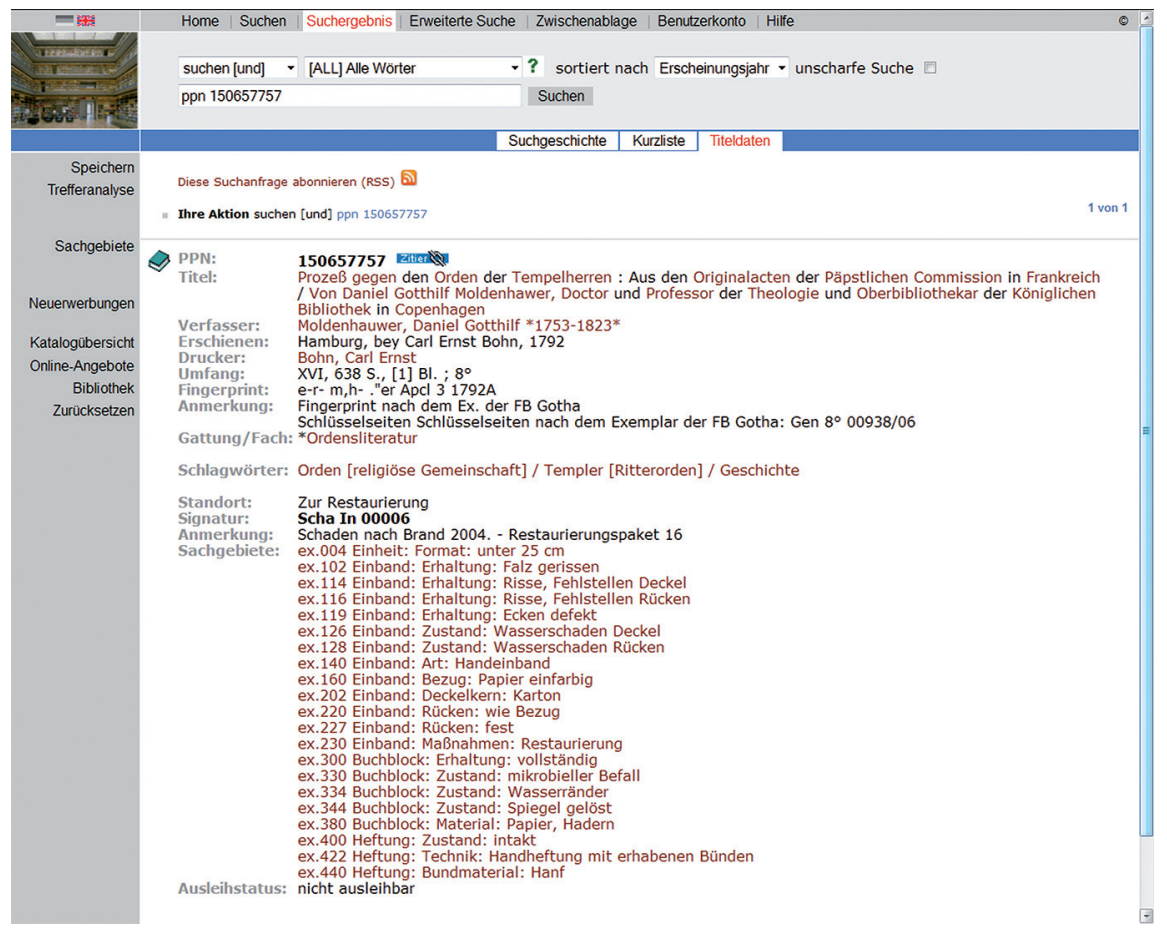

Abb. 1: Schadensdokumentation im OPAC. Im unteren Teil der Aufnahme sind die Ergebnisse der Restauratoren als lokale Normsätze eingetragen.

Eines der Ziele dieser Normierung war, gleichartige Schadensfälle leicht und verlässlich ermitteln zu können, um die dabei gebildeten Teilmengen jederzeit flexibel nach formalen Kriterien der bereits vorhandenen Katalogdaten gruppieren zu können. Es ist daher möglich, in konkreten Zahlen darüber Auskunft zu geben, wie viele Bücher zum Beispiel Schäden wie „defekte Gelenkfunktion“, „lose Deckel“ oder Verschmutzungen aufwiesen; wie viele davon Leder-, Pergament-, Textil- oder Pappeinbände haben, aber zum Beispiel auch wie viele davon aus dem 16., 17. oder 18. Jahrhundert stammen. Insgesamt wurden für die leichten Schäden ca. 16000 und für die gravierenderen Einbandschäden ca. 22000 Einträge vorgenommen.

Weitere Vorzüge der Dokumentationsarbeit innerhalb der Strukturen des Verbundkatalogs bestehen für die Schadensdokumentation in der Möglichkeit, den Status der Restaurierung festzuhalten, die Bände in den etablierten Formen zu verbuchen und den Lesern jederzeit Transparenz in Fragen der Verfügbarkeit der betroffenen Exemplare zu bieten. Es bleibt noch anzumerken, dass nicht alle 
Möglichkeiten, die diese Form der Erschließung bietet, auch für die Praxis der Auftragsvergabe zur Einbandrestaurierung genutzt werden konnten.

\subsection{Dokumentation der sogenannten „Aschebücher“}

Besonders schwierig liegen die Verhältnisse für Restauratoren wie Bibliothekare bei den sogenannten „Aschebüchern“: Erstens führen die Schädigungen (d.h. das Fehlen von intakten Einbänden, die Verschmutzung mit Asche, der fragile feuer- und hitzegeschädigte Seitenrand, die Auswirkungen der mit Löschwasser oder -schaum eingebrachten Substanzen und andere Umstände mehr) zu großen Schwierigkeiten für so grundlegende Vorgänge wie etwa das Herausheben der Bände aus ihrer Verpackung, das Umblättern und das Lesen. Zweitens bewirkte das Vordringen der Schädigung am Buchkörper von der Peripherie zum Zentrum hin, dass außer den Einbänden und Papierrändern (Schnitte) häufig auch die ersten und letzten Seiten fehlen. Diese Seiten sind aber mit Titelblatt, Kupfertitel, Frontispiz oder Widmungen bzw. mit Druckvermerk, Kolophon oder Impressum gerade diejenigen Teile der Bücher, denen ein Großteil der für die Katalogisierung wichtigen Metadaten entnommen werden kann; außerdem tragen diese Teile des Buchkörpers in der Regel die Signatur. Das Fehlen oder die Beeinträchtigung dieser Informationen erschwert also die Formalerschließung oder führt dazu, dass die Werke oder Ausgaben nicht mehr ohne Weiteres zu identifizieren sind. Eine dritte Schwierigkeit besteht darin, dass ursprünglich zusammen gehörende Teile eines Bandes, eines mehrbändigen Werkes oder eines Sammelbandes durch die Widrigkeiten des Brandes selbst, aber auch durch die notdürftige Bergung, die anschließende Gefriertrocknung oder durch das Sortieren, Verpacken und den Transport voneinander getrennt worden sind. 


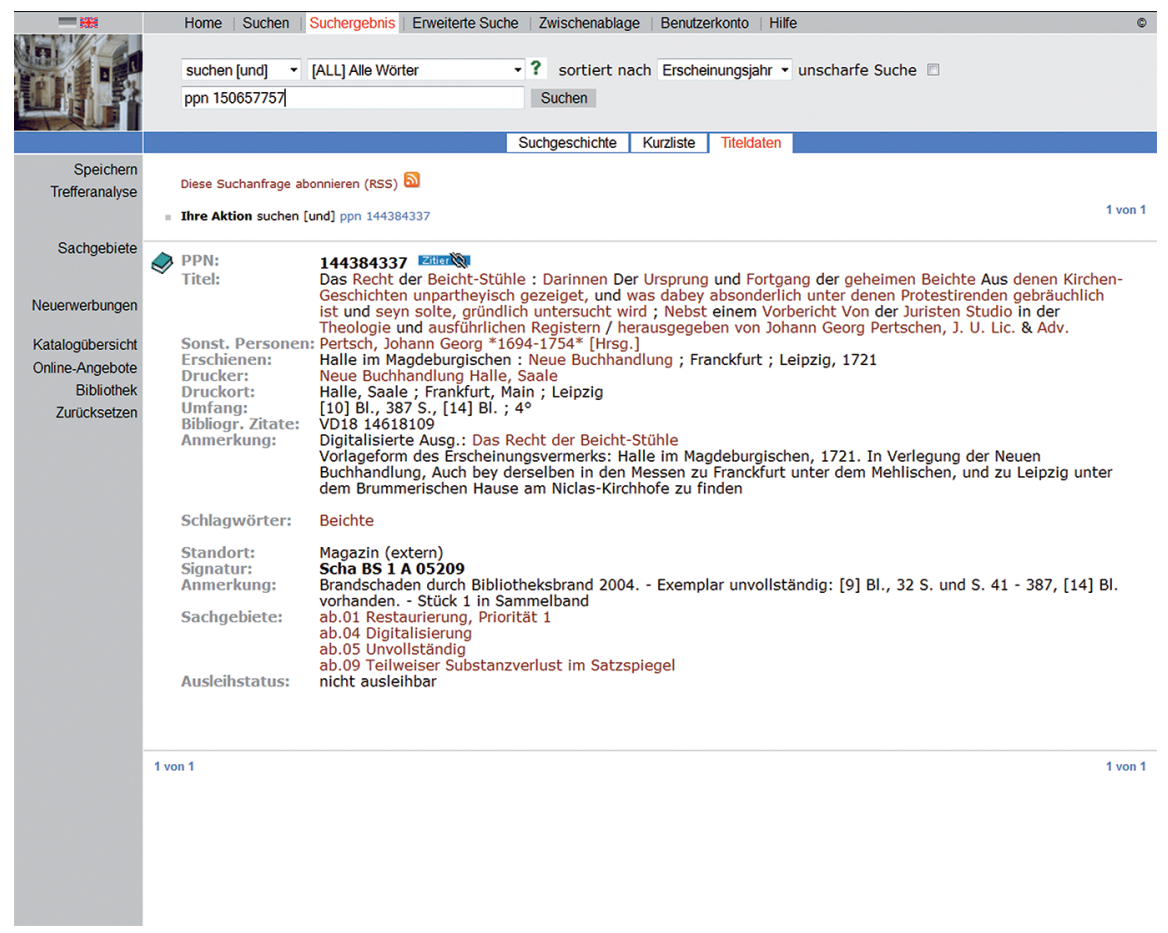

Abb. 2: Dokumentation eines unvollständigen Aschebuchs (OPAC-Ansicht).

Trotz dieser Schwierigkeiten sind inzwischen ca. 1400 Bände bzw. 250000 Blätter in diesem Schadenssegment restauriert worden. Außerdem sind ca. 7000 Titel in etwa 4000 „Aschebüchern“ im GVK entsprechend dokumentiert (darunter auch solche Fälle, für die keine Restaurierung vorgesehen ist).

Ein nicht zu unterschätzender Vorteil besteht unter diesen schwierigen Arbeitsbedingungen in der ansonsten selten so intensiv gepflegten Zusammenarbeit zwischen Restauratoren und Bibliothekaren: Das Know-how der restauratorischen Seite ermöglicht in einem ersten Schritt das angemessene Autopsieren, Öffnen und Umblättern der geschädigten Bestände. In zwei Schritten werden gemeinsam Erfassungsbögen zu den begutachteten Stücken angelegt. Die Kenntnisse der bibliothekarischen Seite sind dabei die Grundlage der strukturierten - soweit möglich normierten - Erfassung der Metadaten (also der Eingaben in den Verbundkatalog), bevor wieder die Professionalität der Restauratoren Reinigung, Stabilisierung und die eigentliche Wiederherstellung ermöglicht. In weiteren anschließenden Schritten sind erneut Kompetenzen aus der Erschließung gefragt: Die Dokumentation der restaurierten Bände im Verbundkatalog wird 
ihrem erneuerten Zustand angepasst, d. h. die vorhandenen Katalogisate werden entsprechend aktualisiert und angereichert. Zuletzt wird in den meisten Fällen die Digitalisierung beauftragt und schließlich auch das Digitalisat im Verbundkatalog erschlossen (dazu ausführlicher unten, Abschnitt 4).

\subsection{Koordination von Restaurierung und Wiederbeschaffung}

Um dublette Erwerbungen zu vermeiden, erfolgt seit 2012 parallel $\mathrm{zu}$ diesen Arbeiten die Durchsicht noch nicht gesichteter „Aschebücher“ sowie der oben beschriebenen Erhebungsbögen durch das bibliothekarische Projekt zur Wiederbeschaffung von Brandverlusten. ${ }^{9}$ Die Befunde dieser Durchsicht werden frühzeitig ebenfalls im Verbundkatalog eingetragen, sind allerdings nur für die Bearbeiter sichtbar. Bislang konnten so bereits ca. 2700 Datensätze markiert werden, wobei in mindestens 900 Fällen die Markierung von „Aschebüchern“ ohne Substanzverlust im Satzspiegel teure Ersatzbeschaffungen überflüssig machte. Dass dieser zusätzliche Aufwand sinnvoll ist, zeigen nicht nur Extrembeispiele für Verluste besonders rarer Drucke wie etwa einer seltenen Kopernikus-Erstausgabe: Ein anderes Exemplar dieser Ausgabe wurde im Juni 2008 von einem Auktionshaus in New York für den Preis von 2,2 Millionen Dollar verkauft. Das Weimarer Stück stand in der Signaturengruppe $4^{\circ}$ XVI auf der Zweiten Galerie des Rokokosaals und gilt zur Zeit noch als Verlust. Während dieses Exemplar unter den „Aschebüchern“ also noch nicht identifiziert werden konnte, sind zahlreiche andere Drucke wie z. B. eine vom Brand betroffene Inkunabel im Rahmen der „Aschebuch“-Bearbeitung wieder aufgefunden worden. ${ }^{10}$

\footnotetext{
9 Zu diesem Projekt: Lorenz, Katja; Mangei, Johannes: Ersatzbeschaffung für Brandverluste der Herzogin Anna Amalia Bibliothek - Routinebetrieb und innovative Verfahren. In: Bibliotheksdienst 43 (2009) S. 978-989 sowie die in Anm. 4 genannte Literatur.

10 Isidorus <Hispalensis>: De summo bono. Köln: Johann Landen, ca. 1496, alte Signatur: $12^{\circ}$ VIII,8 [b], neue Signatur: Scha BS 1 A 00576 (2). GW M15290. Zum Digitalisat: http://opac.ub.uniweimar.de/DB=2/PPNSET?PPN=689167628 [Zugriff: 28. März 2013].
} 


\section{Erschließungsaspekte bei der Digitalisierung der „Aschebücher“}

Zum Workflow der „Aschebuch“-Restaurierung gehört in genau definierten Fällen die Digitalisierung der restaurierten Bände. ${ }^{11}$ Unter dem Blickwinkel der Erschließung sollen im Zusammenhang mit den Digitalisaten hier nur zwei Fragen aufgeworfen werden:

Erstens, wie wird dafür Sorge getragen, dass die Benutzer die Digitalisate auffinden können? Darauf gibt es mehr als eine Antwort, weil auch mehrere verschiedene Zugänge vorgesehen bzw. möglich sind: Zum einen wird - entsprechend den Konventionen des GBV - wie für jedes gedruckte Medium auch für jedes online zugängliche Digitalisat der Sammlung ein Katalogeintrag im Verbundkatalog angelegt. Wenn also nach einem bestimmten Autor oder Titel im Online-Katalog gesucht wird, werden in solchen Fällen zwei Treffer angezeigt: ein Datensatz, der sich auf das Buch, etwa ein „Aschebuch“ bezieht, und ein zweiter, der dessen Digitalisat erschließt. Aus diesem Datensatz wird ein direkter Link zu dem betreffenden Digitalisat im Dokumentenmanagementsystem (DMS) angeboten. ${ }^{12}$ Zum anderen kann jeder Interessierte statt im Katalog auch direkt im DMS recherchieren. Die bibliographischen Daten, nach denen dabei gesucht werden kann, sind eine Teileinspielung der Verbunddaten, die auch dem Online-Katalog zugrunde liegen. Nicht zuletzt werden die Titelangaben auch von den gängigen Internet-Suchmaschinen gefunden, so dass auch auf diesem Wege Zugang zu den Digitalisaten besteht.

Zweitens wäre zu fragen: Wie werden die Bezüge zwischen Digitalisat und Original im Katalog dargestellt? Dazu wurde die Beziehung aus dem Katalog heraus eben schon beschrieben. Zu ergänzen ist noch die Möglichkeit, jederzeit auch vom DMS durch einen Klick direkt zur Anzeige der jeweiligen Datensätze des Originals und des Digitalisats im Online-Katalog zu wechseln. Dabei wird aus der Anzeige des digitalisierten Titels im DMS direkt auf die Anzeige der entsprechenden Kurztitel - ohne weitere Zwischenschritte oder erneute Recherchen verwiesen.

Ein weiterer Erschließungsaspekt in diesem Zusammenhang ist die Vergabe von Strukturdaten im DMS, die das Navigieren innerhalb eines Digitalisats erleichtern. Darauf wird hier jedoch nicht weiter eingegangen, weil diese Form

11 Vgl. den Aufsatz von Kirsten Krumeich im vorliegenden Heft.

12 Dazu der Beitrag von Andrea Heist und Karin Schmidt in diesem Heft. 
der Erschließung nicht im Verbundkatalog, sondern unmittelbar im DMS geleistet wird.

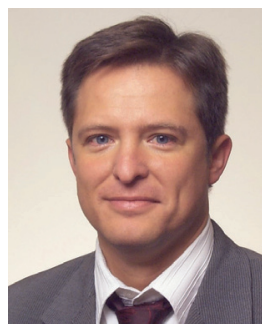

\section{Dr. Johannes Mangei}

Klassik Stiftung Weimar/Herzogin Anna Amalia Bibliothek

Platz der Demokratie 4

D-99423 Weimar

Email: johannes.mangei@klassik-stiftung.de 